\title{
BMJ Open Prevalence and risk factors of depression in patients with lung cancer: protocol for a systematic review and meta-analysis
}

\author{
Xiao-ru Yan, ${ }^{1,2}$ Xun Chen, ${ }^{3,4}$ Peitong Zhang ${ }^{1}$
}

To cite: Yan X, Chen X, Zhang $P$. Prevalence and risk factors of depression in patients with lung cancer: protocol for a systematic review and meta-analysis. BMJ Open 2019:9:e028994. doi:10.1136/ bmjopen-2019-028994

- Prepublication history for this paper is available online. To view these files, please visit the journal online (http://dx.doi. org/10.1136/bmjopen-2019028994).

$\mathrm{X}-\mathrm{Y}$ and $\mathrm{XC}$ contributed equally.

Received 7 January 2019

Revised 15 May 2019

Accepted 11 June 2019

Check for updates

(c) Author(s) (or their employer(s)) 2019. Re-use permitted under CC BY-NC. No commercial re-use. See rights and permissions. Published by BMJ.

${ }^{1}$ Department of Oncology, Guang'anmen Hospital, China Academy of Chinese Medical Sciences, Beijing, China

${ }^{2}$ Tianjin University of Traditional Chinese Medicine, Tianjin, China ${ }^{3}$ Beijing University of Chinese Medicine, Beijing, China

${ }^{4}$ Department of

Gastroenterology, Dongfang Hospital, Beijing University of Chinese Medicine, Beijing, China

Correspondence to

Professor Peitong Zhang;

zhangpeitong@sohu.com

\section{ABSTRACT}

Introduction Patients with lung cancer often experience heavy psychological distress, especially depression, which results in poorer quality of life, shorter survival time and greater mortality. Our aim is to summarise data on the prevalence and risk factors of depression in patients with lung cancer.

Methods and analysis We will search PubMed, EMBASE, MEDLINE, Cochrane Library, Web of Science, China National Knowledge Infrastructure, Wanfang and Chinese Biomedicine Literature Database (SinoMed) for studies on the prevalence and risk factors of depression in patients with lung cancer, which should be published from 1 January 1975 to 25 November 2018 in English/Chinese. Two reviewers will independently screen studies, extract data and assess the risk of bias. We will use RevMan V.5.0 and STATA V.12.0 software for statistical analysis. The $\mathrm{I}^{2}$ test will be used to identify the extent of heterogeneity. Publication bias will be assessed by generating a funnel plot and performing the Begg and Egger test. The quality of the systematic review will be evaluated using the AMSTAR ('A Measurement Tool to Assess Systematic Reviews') criteria and 'The Grading of Recommendations Assessment, Development and Evaluation'.

Ethics and dissemination Since this is a review involving analysis of publicly available data, ethical approval is not required. The final results of this study will be published in a peer-reviewed journal.

PROSPERO registration number CRD42018118167.

\section{INTRODUCTION}

Lung cancer is one of the most frequent cancer diagnoses and has been the leading cause of cancer-related mortality. Lung cancer accounted for $11.6 \%$ of all new cancer cases and $18.4 \%$ of all cancer mortality in 2018 . Lung cancer-related mortality around the world is expected to grow up to three million until $2035 .^{2}$ The majority of lung cancers are diagnosed at a late stage, and the 5-year survival rate for patients with distant stage is only $4 \%$ even after receiving many therapies. ${ }^{3}$ Fear of death, both disease-specific and treatment-induced symptoms, inefficacy of therapy, as well as the expensive cost result in

\section{Strengths and limitations of this study}

Eight databases and grey literature will be searched in this study.

- This review will include cross-sectional, cohort and case-control studies to assess the prevalence and risk factors of depression in patients with lung cancer.

- This study will be conducted in strict accordance with the guidelines of the Preferred Reporting Items for Systematic Review and Meta-Analysis and the Meta-analysis of Observational Studies in Epidemiology.

- A largely sensitive search strategy and strict screening process will be performed to obtain the maximum available studies.

- Some applicable data may be lost as the language in this review will be restricted to English and Chinese.

patients with lung cancer experiencing more considerable psychological distress, particularly depression, compared with the general population. ${ }^{4}$ Related studies have shown that the incidence of depression among patients with lung cancer ranged from $12.4 \%$ to $65 \%$. Considering such a high prevalence, attention has turned to explore the effects of depression on patients with lung cancer and its risk factors.

Patients with lung cancer with concomitant depression have worse quality of $\operatorname{life}^{56}$ and longer hospital stay. ${ }^{7}$ One study has revealed that depression was independently associated with poor treatment adherence and unfavourable prognosis. ${ }^{8}$ Moreover, depressive symptoms in patients with lung cancer could lead to increased mortality. ${ }^{9}$ In view of such serious impact of depression on patients with lung cancer, early screening for depression is quite essential. However, investigating every patient is challenging to accomplish. As a consequence, identifying high-risk groups is of tremendous assistance. 
Although a number of researchers have found a variety of factors that can influence the prevalence of depression, such as pain, gender, age, residence, marital status, education level, pathological type, cancer stage and personality characteristics, ${ }^{810-13}$ some of the results are inconsistent. For example, several studies suggest that being female is an independent risk factor for depression in patients with lung cancer, ${ }^{814}$ while others have found gender is not associated with depression. ${ }^{10}{ }^{15}$ Part of the studies demonstrate that older ${ }^{15} /$ younger $^{14}$ age is an independent risk factor. However, some researchers present that age is not a predicting factor for depression. ${ }^{10}{ }^{16}$ Only after identifying high-risk patients can we offer more targeted treatment strategies for them. Therefore, we decided to conduct a systematic review and meta-analysis to provide robust evidence on the risk factors related to depression and put forward some appropriate treatment suggestions. The findings of this study would assist clinicians in identifying patients with lung cancer with depression and provide evidence for designing early targeted interventions for high-risk survivors that can attenuate these negative emotional reactions.

\section{Objective}

Our aim is to assess the prevalence and risk factors of depression in patients with lung cancer.

\section{METHODS AND ANALYSIS}

This protocol has been designed according to the guidelines of the Preferred Reporting Items for Systematic Review and Meta-Analysis Protocols ${ }^{17}$ and the Meta-analysis of Observational Studies in Epidemiology. ${ }^{18}$ This systematic review and meta-analysis has been registered with the International Prospective Register of Systematic Reviews. The protocol began on 1 December 2018 and is expected to be completed on 30 December 2019.

\section{Patient and public involvement}

Since this review will be based on published studies, there will be no patient or public involvement.

\section{Study design and participants}

Inclusion criteria

Observational studies with available data on the prevalence and risk factors associated with depression among patients with lung cancer will be considered. We will include cross-sectional, cohort and case-control studies (with a sample of at least 30). Articles should have been published in English/Chinese in indexed and peer-reviewed journals from 1 January 1975 to 25 November 2018.

\section{Type of participants}

Participants will be patients with lung cancer.

\section{Exclusion criteria}

- Case reports, case series, opinion papers, qualitative research, letters to the editor, comments, conference proceedings, policy papers, reviews and meta-analyses, study protocols without baseline data, and animal studies will be excluded.

- For duplicate studies, the most comprehensive and/ or recent study with the largest sample size will be considered.

- Studies with incomplete data, even after requesting from the corresponding author, will be excluded. For example, studies without complete baseline data, or without OR value, or where OR value and $95 \%$ CI cannot be calculated will not be included.

\section{Outcomes}

- Prevalence of depression among patients with lung cancer.

- Risk factors associated with depression in patients with lung cancer.

- The strength of association between each risk factor and depression.

\section{Search methods for identification of relevant studies Electronic searches}

The following databases will be searched: PubMed, EMBASE, MEDLINE, Cochrane Library, Web of Science, China National Knowledge Infrastructure, Wanfang and Chinese Biomedicine Literature Database (SinoMed). The reference lists of the selected studies and review articles will also be manually screened to identify any additional relevant articles. The search strategy will be developed with the assistance of an experienced librarian.

\section{Search strategy}

The search will be a combination of the following medical subject headings terms and free words.

- Participants (lung cancer/lung neoplasms/lung carcinoma/pulmonary neoplasms/pulmonary cancer).

- Interventions (risk factors).

- Outcomes (depression/depressive symptom/depressive disorder/depressed). Depression is defined by different widely accepted scales, such as the Beck Depression Inventory; International Classification of Diseases, Tenth Revision; Hospital Anxiety and Depression Scale-Depression; Diagnostic and Statistical Manual of Mental Disorders, Fourth Edition; Hamilton Depression Scale; and Self-Rating Depression Scale.

- Study design (cross-sectional study/cohort study/ case-control study).

Moreover, Google Scholar will be used to search for grey literature. If the full text of an article cannot be found, the corresponding author will be contacted through email. If the corresponding authors do not respond after multiple attempts, the study will be excluded. The details of our search strategy for PubMed are presented in box 1 .

\section{Screening for studies}

Studies imported into EndNote V.X8 after removing duplicates will be independently reviewed by two authors (X-RY and $\mathrm{XC}$ ) based on the exclusion and 


\section{Box 1 Main search strategy for PubMed}

((Lung Cancer[MeSH]OR Lung Cancer[Text Word]OR lung neoplasms[MeSH] OR lung neoplasms [Text Word]OR lung carcinoma [Text Word]OR Pulmonary Neoplasms[Text Word] OR Pulmonary cancer[Text Word])AND(Depression[MeSH] OR Depression* Text Word]OR Depressive Symptom*[Text Word]OR Symptom*, Depressive[Text Word] OR Depressive Disorder[MeSH]OR Depressive Disorder*[Text Word]OR Disorder*, Depressive[Text Word] OR depressed[MeSH] OR depressed[Text Word])AND(Risk Factors[MeSH]OR Risk Factor*[Text Word]OR Factor ${ }^{\star}$, Risk[Text Word] OR factor*[Text Word] OR influence[Text Word] OR impact*[Text Word])AND(prevalence[MeSH] OR frequency[MeSH] OR cross-sectional study[MeSH] OR cross-sectional[title/abstract] OR retrospective*[title/abstract] $\mathrm{OR}$ registry[title/abstract] $\mathrm{OR}$ consecutive*[title/abstract] OR prospective*[title/abstract] OR case-control[title/ abstract] OR controlled trial[title/abstract]) NOT (Meta analysis[title] OR 'systematic review'[Title] OR review[title/abstract])) Publication date limits: from 1 January 1975 to 25 November 2018 Language limits: English/Chinese

inclusion criteria. First, they need to screen the titles and abstracts to select potentially related studies. If there is any uncertainty, the article will be included in the next stage. Then the full text of the selected studies will be assessed for final eligibility. If the two authors cannot reach agreement on articles for inclusion, a third author (PZ) will decide whether to include or exclude the article.

\section{Data extraction}

All data will be extracted and recorded independently by two reviewers (X-RY and XC) in an electronic database created in Microsoft Office Excel V.2010. The results (titles and/or abstracts) from the databases will be merged using EndNote V.X8 to facilitate the removal of duplicates. Disagreements will be resolved by discussion or with the verdict of a third author (PZ). Any unclear information from an included article will be clarified after contacting the corresponding authors, or only available data will be analysed. The information that will be extracted include the first author's name, date of publication, journal, type of study (cross-sectional/cohort/casecontrol), country and region, sample size ( $\mathrm{N}$ and male/ female), duration of the study, baseline age, diagnostic criteria for lung cancer and depression, incidence of depression/mean and SD for depression score, variables, OR values, $95 \% \mathrm{CI}$, and other relevant data for quality evaluation and risk of bias assessment. Furthermore, the reasons for exclusion of studies while extracting will also be recorded. Possible risk factors include but are not limited to gender, age, occupation, marital status, education level, social support, alcohol status, smoking status, pathological type, cancer clinical stage, Karnofsky Performance Status (KPS), disease course and therapy method. The variables extracted will be adjusted in the process as it is likely that more and more variables that need to be included will turn up.

\section{Assessment of risk of bias in the included studies}

The risk of bias/method quality of the included studies will be assessed independently by two authors (X-RY and $\mathrm{XC}$ ) at the study and outcome levels. Any disagreements will be solved by discussion or with arbitrament of the third author (PZ). In this study, we will use the Newcastle-Ottawa Scale to evaluate the quality of studies. This scale is a non-randomised controlled trial quality evaluation instrument with scores ranging from 0 to 9 ; scores of 0-4 and 5-9 mean low quality and high quality, respectively. ${ }^{19}$

\section{Data analysis}

We will use RevMan V.5.0 and STATA V.12.0 software for analysis. Meta-analysis will be performed when the heterogeneity is low or the source could be found, although heterogeneity is high. A systematic narrative synthesis will be conducted if it is impossible to complete any meta-analysis. RevMan V.5.0 will be used to calculate the OR values and 95\% CIs of the reported risk factors for depression in lung cancer. When the CI of the OR value is not equal to 1 and $p<0.05$, we believe it is statistically significant.

The prevalence estimates reported by the individual studies will be extracted or converted into per cent prevalence, and their respective SEs will be calculated. For the depression scores, Standardized Mean Difference (SMD) will be used for analysis. We will use the Freeman-Tukey double arcsine transformation to stabilise the variance of study-specific prevalence. The prevalence of each study will be recalculated to confirm numerators and denominators, and adjustments will be made if necessary. The $\mathrm{I}^{2}$ test will be used to identify the extent of heterogeneity. When the $\mathrm{I}^{2}$ value is less than $50 \%$, the fixed-effects model will be used. If the $\mathrm{I}^{2}$ value is higher than $50 \%$, the random-effects model will be used, because we think the results of each study vary markedly. In this study, factors of high heterogeneity will be removed one by one to find the source of any observed heterogeneity. The causes of heterogeneity may include differences in study design, statistical methods and participants. When the factor impacts the results largely, it will be removed.

Subgroup analysis will be done when data are available. The groups may be designed by country or region, diagnostic criteria for depression, bias score, time since diagnosis (long-term vs short-term survivors), severity/staging of lung cancer and study design.

We will assess publication bias by generating a funnel plot and performing the Begg and Egger test $(\mathrm{p}<0.05$ indicates the existence of publication bias).

\section{Quality control of the systematic review and meta-analysis}

The methodological quality of the systematic review will be evaluated using the AMSTAR (A Measurement Tool to Assess Systematic Reviews) criteria. ${ }^{20}$ The Grading of Recommendations Assessment, Development and Evaluation will also be used to evaluate the strength of evidence produced by the systematic review. 


\section{ETHICS AND DISSEMINATION}

The final results of this study will be published in a peer-reviewed journal. This systematic review and meta-analysis for assessing the risk factors related to depression in patients with lung cancer would provide evidence for designing early targeted interventions for high-risk survivors that can attenuate negative emotional reactions.

Contributors $\mathrm{PZ}, \mathrm{X}-\mathrm{RY}$ and $\mathrm{XC}$ conceived the study. $\mathrm{X}-\mathrm{RY}, \mathrm{XC}$ and $\mathrm{PZ}$ designed the study protocol. $X-R Y$ and $X C$ drafted the initial manuscript. $P Z$ revised the protocol. $\mathrm{PZ}$ is the guarantor of the review. All authors read and approved the final version of the manuscript prior to submission.

Funding This review will be supported by the 'National Natural Science Foundation of China' (no 81673797) and the 'Beijing Municipal Natural Science Foundation' (no 7182142).

Competing interests None declared.

Patient consent for publication Not required.

Ethics approval Since this is a review involving analysis of publicly available data, ethical approval is not required.

Provenance and peer review Not commissioned; externally peer reviewed.

Open access This is an open access article distributed in accordance with the Creative Commons Attribution Non Commercial (CC BY-NC 4.0) license, which permits others to distribute, remix, adapt, build upon this work non-commercially, and license their derivative works on different terms, provided the original work is properly cited, appropriate credit is given, any changes made indicated, and the use is non-commercial. See: http://creativecommons.org/licenses/by-nc/4.0/.

\section{REFERENCES}

1. Bray F, Ferlay J, Soerjomataram I, et al. Global cancer statistics 2018: GLOBOCAN estimates of incidence and mortality worldwide for 36 cancers in 185 countries. CA Cancer J Clin 2018;68:394-424.

2. Didkowska J, Wojciechowska U, Mańczuk M, et al. Lung cancer epidemiology: contemporary and future challenges worldwide. Ann Transl Med 2016;4:150.

3. Miller KD, Siegel RL, Lin CC, et al. Cancer treatment and survivorship statistics, 2016. CA Cancer J Clin 2016;66:271-89.

4. Haun MW, Sklenarova H, Villalobos M, et al. Depression, anxiety and disease-related distress in couples affected by advanced lung cancer. Lung Cancer 2014;86:274-80.
5. Polański J, Chabowski M, Chudiak A, et al. Intensity of Anxiety and Depression in Patients with Lung Cancer in Relation to Quality of Life. Adv Exp Med Biol 2018;1023:29-36.

6. Choi S, Ryu E. Effects of symptom clusters and depression on the quality of life in patients with advanced lung cancer. Eur $J$ Cancer Care 2018;27:e12508.

7. Gu W, Xu YM, Zhong BL. Health-related quality of life in Chinese inpatients with lung cancer treatedin large general hospitals: acrosssectional study. BMJ Open 2018;8:e019873.

8. Arrieta $\mathrm{O}$, Angulo LP, Núñez-Valencia $\mathrm{C}$, et al. Association of depression and anxiety on quality of life, treatment adherence, and prognosis in patients with advanced non-small cell lung cancer. Ann Surg Oncol 2013;20:1941-8.

9. Sullivan DR, Forsberg CW, Ganzini L, et al. Longitudinal Changes in Depression Symptoms and Survival Among Patients With Lung Cancer: A National Cohort Assessment. J Clin Oncol 2016;34:3984-91.

10. Park $\mathrm{S}$, Kang $\mathrm{CH}$, Hwang $\mathrm{Y}$, et al. Risk factors for postoperative anxiety and depression after surgical treatment for lung cancert. Eur $J$ Cardiothorac Surg 2016;49:e16-e21.

11. Shimizu K, Nakaya N, Saito-Nakaya K, et al. Clinical biopsychosocial risk factors for depression in lung cancer patients: a comprehensive analysis using data from the Lung Cancer Database Project. Ann Oncol 2012;23:1973-9.

12. Gu W, Xu YM, Zhu JH, et al. Depression and its impact on healthrelated quality of life among Chinese inpatients with lung cancer. Oncotarget 2017;8:104806-12.

13. Andrykowski MA, Steffens RF, Bush HM, et al. Disparities in mental health outcomes among lung cancer survivors associated with ruralness of residence. Psychooncology 2014;23:428-36.

14. Sullivan DR, Forsberg CW, Ganzini L, et al. Depression symptom trends and health domains among lung cancer patients in the CanCORS study. Lung Cancer 2016;100:102-9.

15. Hong JS, Tian J. Prevalence of anxiety and depression and their risk factors in Chinese cancer patients. Support Care Cancer 2014;22:453-9.

16. Shi Y, Gu F, Hou LL, et al. Self-reported depression among patients with non-small cell lung cancer. Thorac Cancer 2015;6:334-7.

17. Shamseer L, Moher D, Clarke M, et al. Preferred reporting items for systematic review and meta-analysis protocols (PRISMA-P) 2015: elaboration and explanation. BMJ 2015;349:g7647-g47.. (jan02 1):

18. Stroup DF, Berlin JA, Morton SC, et al. Meta-analysis of observational studies in epidemiology: a proposal for reporting. Meta-analysis Of Observational Studies in Epidemiology (MOOSE) group. JAMA 2000;283:2008-1.

19. Stang A. Critical evaluation of the Newcastle-Ottawa scale for the assessment of the quality of nonrandomized studies in metaanalyses. Eur J Epidemiol 2010;25:603-5.

20. Shea BJ, Grimshaw JM, Wells GA, et al. Development of AMSTAR: a measurement tool to assess the methodological quality of systematic reviews. BMC Med Res Methodol 2007;7:10. 